Running head: TRANSFER OF ATTENTIONAL RETRAINING

\title{
Limited Transfer of Threat Bias Following Attentional Retraining
}

Bram Van Bockstaele ${ }^{\mathrm{a}}$, Ernst H. W. Koster ${ }^{\mathrm{a}}$, Bruno Verschuere ${ }^{\mathrm{a}, \mathrm{b}, \mathrm{c}}$, Geert Crombez ${ }^{\mathrm{a}}$, and Jan De Houwer ${ }^{\mathrm{a}}$

a Department of Experimental Clinical and Health Psychology, Faculty of Psychology and Educational Sciences, Ghent University

b Department of Clinical Psychology, Faculty of Social and Behavioural Sciences, University of Amsterdam

c Faculty of Psychology and Neurosciences, Maastricht University, The Netherlands

Corresponding author:

Bram Van Bockstaele

Department of Experimental Clinical and Health Psychology

Faculty of Psychology and Educational Sciences

Ghent University

Henri Dunantlaan 2

B-9000 Ghent, Belgium

Email: Bram.Vanbockstaele@UGent.be

Bram.Vanbockstaele@gmail.com 


\begin{abstract}
Background and objectives. Anxiety-related attentional bias for threat is considered an important risk factor for the development and maintenance of anxiety disorders. In line with this idea, recent studies have illustrated that experimentally induced changes in attentional bias have an impact on both non-clinical and clinical levels of anxiety. Still, little is known about the potential transfer of computerized training of attention to different components of attentional processing of threat.

Methods. In the present study, we trained participants to either avoid or attend towards threatening pictures in a dot probe task, and we examined whether this attentional training transferred to a measure of emotional interference.
\end{abstract}

Results. Despite our successful manipulation of attentional bias in the dot probe task, we found no generalization of the attentional training to the interference task.

Limitations. It is possible that our study lacked statistical power to reveal possible group differences in the interference task.

Conclusions. Our study shows that attentional training using the dot probe task may influence the amount of attention that is given to the spatial location of threat, but not necessarily the amount of attention that is given to the semantic content of stimuli.

Keywords: attentional bias, training, threat, anxiety, transfer 


\section{Limited Transfer of Threat Bias Following Attentional Retraining}

A basic tenet of several cognitive theories of anxiety disorders is that certain information processing biases are at the core of fear and anxiety disorders (e.g., Beck, Emery, \& Greenberg, 1985; Eysenck, 1992; Mogg \& Bradley, 1998; Williams, Watts, MacLeod, \& Mathews, 1997). For instance, Williams et al. suggested that anxious individuals are more likely to interpret ambiguous events as threatening (interpretation bias), to recall threatening episodes from memory (memory bias), and to orient to threatening information in their environment (attentional bias). Importantly, these processing biases have been argued to be not just epiphenomena of fear and anxiety, but may be causally involved in the aetiology or maintenance of fear and anxiety (e.g., Williams et al.).

A crucial test of this causality assumption was provided by MacLeod, Rutherford, Campbell, Ebsworthy and Holker (2002), who trained participants to either avoid or attend to threatening words using a dot probe paradigm (MacLeod, Mathews, $\&$ Tata, 1986). In this paradigm, two cues (typically one threatening and one neutral stimulus) are presented on different spatial locations, and participants are required to respond as quickly and as accurately as possible to the location or the identity of the target. An attentional bias towards threatening stimuli is inferred from faster responses on trials where the target appears on the same location as the threatening cue (congruent trials) compared to trials where the target appears on the location of the neutral cue (incongruent trials). In order to change attentional bias, MacLeod et al. (2002) manipulated the proportion of congruent and incongruent trials between participants. In an attend threat group, they presented more congruent trials, whereas in an avoid threat group, they presented more incongruent trials. After this attentional training phase, 
participants who were trained to avoid threat experienced less emotional distress during a stressful task compared to participants who were trained to attend to threat. This result has inspired researchers to investigate the clinical application of computerized attention training to reduce anxiety symptoms. Recently, experimentally induced reductions in attentional bias using the modified dot probe task have been shown to reduce symptoms of social anxiety (Amir, Weber, Beard, Bomyea, \& Taylor, 2008; Amir, Beard, Taylor et al., 2009; Li, Tan, Qian, \& Liu, 2008; Schmidt, Richey, Buckner, \& Timpano, 2009) and generalized anxiety disorder (Amir, Beard, Burns, \& Bomyea, 2009; Hazen, Vasey, \& Schmidt, 2009). As such, the reduction of attentional bias seems to be effective as a clinical intervention for anxiety disorders (e.g., Hakamata et al., 2010).

Despite the clinical potential of attentional bias training for anxiety disorders, some reservations need to be taken into consideration. First, attentional bias training seems not to be effective for all anxiety disorders. For instance, Reese, McNally, Najmi and Amir (2010) found no differences in spider fear between participants who were trained to avoid spiders and participants in a no-training control group (see also Van Bockstaele et al., 2011). Second, although attentional bias modification is effective in reducing symptoms of anxiety, we know little about the processes through which this therapeutic effect is accomplished. One such mechanism may be generalization. Indeed, it is often implicitly assumed that the training for a specific set of threatening stimuli generalizes to other stimuli. That is, it is assumed that after training the individual will avoid all threatening stimuli, and not only the stimuli that were used during training. Likewise, it is often implicitly assumed that the attentional effects obtained after training with the dot probe task are not restricted to that particular paradigm, but also may be found in other situations or paradigms. Noteworthy, the selection of the dot 
probe task as a training procedure has mainly been based on practical considerations (see Mathews \& MacLeod, 2002), and there is yet little research on whether the modified dot probe task is suitable to change attentional functioning in everyday life. As such, the applied potential of the modified dot probe task as an attentional training task depends on how well the obtained attentional training effect transfers to other tasks, settings, and stimuli. At present, this feature of the modified dot probe task has received only limited study. In two studies, Amir and colleagues (Amir et al., 2008, Amir, Beard, Taylor et al., 2009) found that attentional training with the dot probe task generalized to attentional bias for threatening words as measured with a spatial cueing task. However, the spatial cueing task is highly similar to the dot probe task so this effect only suggests that there is transfer of training to conditions that very closely resemble the training conditions (close transfer). To date, more broad levels of transfer of training have not been scrutinized.

In the present study, we investigated transfer of training of two different attentional manipulations in the dot probe task on emotional interference. Emotional interference was assessed both within the dot probe task and in a separate interference task, similar to the one used by Mogg, Garner, Holmes, and Bradley (2008). We trained one group of participants to attend towards threatening pictures, whereas we trained another group of participants to avoid threat. This training was preceded and followed by a standard version of the dot probe task to investigate whether training was successful in changing attentional bias, and whether the training led to reduced interference within the dot probe task. After the attentional training phase, participants completed an emotional interference task with a new set of pictures. In this task, a single stimulus, either threatening or neutral, preceded the presentation of a target. 
Typically, participants are slower to respond to the target if it was preceded by an emotional picture relative to a neutral picture (e.g., Schimmack, 2005). The underlying mechanism of the interference effect has been related a difficulty to disengage attention away from threatening stimuli (e.g., Fox, Russo, Bowles, \& Dutton, 2001). According to this view, the irrelevant emotional dimension of the cue holds attention, which in turn impairs the processing of other, task-relevant stimuli and thus delays the appropriate response (see also De Houwer \& Tibboel, 2010). If the interference effect is based on such difficulty to disengage attention away from threat, the attentional training manipulation should have a clear impact on this effect. More specifically, as participants in the avoid threat group are trained to disengage their attention away from threat, they should be less influenced by the threatening cues in the interference task, leading to a smaller interference effect. Finally, after the interference task, participants rated all stimuli for arousal, valence and threat value. In general, fear and anxiety are emotions that are marked by high levels of arousal and negative affect (e.g., Rachman, 1998). Therefore, one can hypothesize that a therapeutic intervention that is supposed to reduce fear and anxiety - such as the attentional bias modification procedure - might also reduce these feelings of arousal and negative affect.

$$
\text { Method }
$$

\section{Participants}

Fifty-nine first-year students $(21$ men, average age $=20.54$ years old, $S D=2.96$ ) participated in the experiment as a partial fulfilment of course requirements. All participants signed an informed consent form prior to the beginning of the experiment. Apparatus and Materials 
The entire experiment was programmed using the Inquisit Millisecond 2.0 (2007) software package, and it was run on a Dell Optiplex GX520 desktop computer with a $100 \mathrm{~Hz} 19$-inch colour monitor. We selected 24 pictures from the International Affective Picture System (IAPS; Lang, Bradley, \& Cuthbert, 2005; pictures of the IAPS have been validated for the current population, Verschuere, Crombez, \& Koster, 2001). ${ }^{1}$ Of these 24 pictures, 12 were threatening and 12 were neutral. We divided the threatening pictures in two subsets, matched for valence and arousal (IAPS ratings: valence subset $1=2.98, S D=0.74$, valence subset $2=2.67, S D=0.79$, arousal subset 1 $=6.83, S D=0.37$, arousal subset $2=6.90, S D=0.27 ; t \mathrm{~s}<1)$. Likewise, the neutral pictures were also divided in two subsets (IAPS ratings: valence subset $1=4.95, S D=$ 0.45 , valence subset $2=5.00, S D=0.36$, arousal subset $1=2.86, S D=0.52$, arousal subset $2=2.93, S D=0.72 ; t \mathrm{~s}<1)$. For both threatening and neutral pictures, one subset was used in the dot probe task, and the other was used in the interference task. The assignment of different subsets of pictures to either the dot probe task or the interference task was counterbalanced across participants. Finally, we selected an extra set of six neutral pictures (IAPS ratings: valence $=5.30, S D=0.51$, arousal $=2.68, S D=0.32$ ) for practice trials and neutral dot probe trials (see below).

\section{Questionnaires}

We used the Dutch translations of the state and trait versions of the State and Trait Anxiety Inventory (STAI-S and STAI-T: Spielberger, Gorsuch, Lushene, Vagg, \& Jacobs, 1983; van der Ploeg, Defares, \& Spielberger, 1980) to assess our participants' levels of anxiety. Both questionnaires consist of 20 items, and each item is scored on a four-point Likert scale. The STAI-S assesses the individual's present level of anxiety, whereas the STAI-T measures a more general susceptibility to experience emotional 
distress over an extended period of time. Alpha coefficients in the present study were .88 for the STAI-S and .93 for the STAI-T.

\section{Dot Probe Task}

The dot probe task contained four different trial types. On congruent trials, the target was presented on the location that was cued with a threatening picture. On incongruent trials, the target appeared on the location of the neutral picture. On neutral trials, both cue pictures were neutral, and the target could follow on either location. Finally, we encouraged participants to focus on the centre of the screen by presenting digit trials. On these trials, the fixation cross was replaced by a digit ranging from one to three after $1000 \mathrm{~ms}$. This digit remained on the screen for $100 \mathrm{~ms}$. Participants were required to indicate which digit they had seen by pressing the corresponding key on the upper left of a standard AZERTY keyboard. Participants were required to guess if they were unsure about the answer. In this manner, poor performance on digit trials indicates poor motivation of the participant or a lack of fixation on the fixation cross.

All stimuli in the dot probe task were presented on a black background. Each trial started with the presentation of a fixation cross in the centre of the screen, and two grey rectangles, one above and one below the fixation cross. These rectangles were 4 $\mathrm{cm}$ high by $5.3 \mathrm{~cm}$ wide, and the distance between the centre of the rectangles and the fixation cross was $4 \mathrm{~cm}$. All cues and targets were presented in the centre of the grey rectangles. In congruent and incongruent trials, the cue pairs consisted of one randomly selected threatening picture and one randomly selected neutral picture, whereas on neutral trials, both pictures were neutral. The pictures were adjusted to fit the size of the grey rectangles. The pictures were presented for $500 \mathrm{~ms}$, and were replaced by a $20 \mathrm{~ms}$ grey mask. Next, a target stimulus appeared. Targets consisted of either the letter ' $F$ ' or 
the letter ' $E$ ', and remained on the screen until a response was given. Participants responded by pressing either the ' 1 ' or the ' 3 ' key of the numeric pad of the keyboard with the index or middle finger of their dominant hand, and the next trial started $500 \mathrm{~ms}$ after a response was given. Targets were presented equally often above as below the fixation cross, and were equally often an $\mathrm{E}$ as an $\mathrm{F}$. The assignment of the response keys to the targets was counterbalanced across participants.

The dot probe task consisted of four different phases. First, participants completed a practice phase consisting of 24 neutral trials and 3 digit trials. In this phase, an error message appeared on incorrect responses. The data of this phase were not analysed. The second phase was the pre-training assessment phase. This phase consisted of 48 congruent trials, 48 incongruent trials, 24 neutral trials and 6 digit trials. The third phase was the attentional training phase. In this phase, participants were randomly assigned to one of two groups. In the attend threat group, we presented three blocks consisting each of 96 congruent trials, 24 neutral trials and 6 digit trials. In the avoid threat group, we also presented three blocks, each consisting of 96 incongruent trials, 24 neutral trials and 6 digit trials. The fourth and last phase was the post-training assessment phase, which was identical to the pre-training assessment phase. This last phase allowed us to investigate whether our manipulation of attention successfully induced an attentional bias towards versus away from threat.

\section{Interference Task}

Each trial in the interference task started with the presentation of as single grey rectangle ( $5.3 \mathrm{~cm}$ wide by $4 \mathrm{~cm}$ high) in the centre of the screen, and a black fixation cross in the centre of this rectangle. After $1000 \mathrm{~ms}$, the fixation cross was randomly replaced by either a threatening or a neutral picture. All pictures were adjusted to the 
same size of the grey rectangle. The picture remained on the screen for $200 \mathrm{~ms}$, and was replaced by a grey mask for $50 \mathrm{~ms}$. Then, a target appeared in the centre of the screen. The target was either an ' $\&$ ' or an '@', and participants were required to identify the target as fast and as accurately as possible by pressing the 'W' or the '?' key of a standard AZERTY keyboard with their left or right index finger respectively. Both targets were presented equally often, and the assignment of the response keys to specific targets was counterbalanced across participants. The intertrial interval varied randomly between 500 and $1050 \mathrm{~ms}$.

The interference task consisted of two phases. First, in order to acquaint participants with the task at hand, they completed a practice phase. In this phase, we used the same pictures as the ones that we used in the neutral dot probe trials. The practice phase consisted of 24 trials, and an error message was presented on incorrect responses. The data of the practice phase were not analysed. The second phase was the test phase, which consisted of two identical blocks. In each block, each picture was presented eight times, for a total of 96 trials per block. No error messages were presented on incorrect responses in the test blocks.

\section{Picture Ratings}

After the interruption task, participants were shown all pictures that were used in the experiment (12 threatening and 12 neutral), except the six neutral pictures from the practice phases and the neutral dot probe trials. Each picture was presented against a black background, and participants could look at the picture for as long as they wanted. In order to proceed to the rating screen, participants pushed the space bar. We recorded the picture viewing time as the time between the onset of the pictures and the bar-press. Lang, Greenwald, Bradley and Hamm (1993) showed that people look longer at 
arousing pictures than neutral pictures, and as such, viewing time can be used as an indicator of the arousal of the picture. Upon pressing the space bar, the picture disappeared and participants rated on three separate nine-point Likert scales how threatened $(1=$ "not threatened at all" through $9=$ "very threatened"), aroused $(1=$ "calm, relaxed" through $9=$ "very aroused") and positive or negative ( 1 = "positive" through $9=$ "negative") they felt while viewing the picture.

\section{General Procedure}

The experiment was conducted in a dimly lit room, and participants were seated approximately $50 \mathrm{~cm}$ from the computer screen. First, they completed the STAI-S and the STAI-T. Next, they practiced the dot probe task and their pre-training attentional bias was assessed. Upon completion of the pre-training attentional bias assessment block, participants were randomly allocated to either the attend threat group or the avoid threat group and completed the corresponding version (attend threat versus avoid threat) of the attentional training task, followed by the post-training attentional bias assessment block and the interference task. Finally, they rated the threat value, arousal and valence of all experimental pictures and they again completed the STAI-S.

$$
\text { Results }
$$

\section{Group Characteristics}

At the beginning of the experiment, average trait anxiety was $35.14(S D=8.59)$ and average state anxiety was $31.46(S D=5.73)$. The two groups did not differ on either trait or state anxiety, both $F \mathrm{~s}<1$, both $p \mathrm{~s}>.46$.

\section{Reaction Time Data}

Data reduction and outlier analysis. For the dot probe task, we first removed all trials with errors $(3.40 \%)$. Next, we trimmed the data by removing reaction times faster 
than $150 \mathrm{~ms}$ and slower than $1500 \mathrm{~ms}(0.48 \%)$. Individual outliers were defined as reaction times deviating more than three SDs from the individuals' mean, and were also removed $(1.59 \%)$. We did not analyse the data of neutral trials and the data of the attentional training blocks, because these data do not allow for the calculation of attentional bias scores. Two participants performed poorly on digit trials (participants' scores $=77 \%$ and $80 \%$ correct, group mean $=96 \%$ correct, $S D=5.06$ ), indicating a lack of focus on the fixation cross. Furthermore, two participants made many errors overall (participants' scores $=86 \%$ and $87 \%$ correct, group mean $=97 \%$ correct, $S D=2.62$ ), and one participant was overall very slow (participant's mean latency $=897 \mathrm{~ms}$, group mean $=594 \mathrm{~ms}, S D=78.73$ ). The data of these five participants were not further analysed. For the remaining 54 participants, we calculated attentional bias scores for both the baseline phase and the test phase by subtracting the mean reaction time on congruent trials from the mean reaction time on incongruent trials. Positive attentional bias scores indicate an attentional bias towards threatening stimuli, whereas negative attentional bias scores indicate attentional avoidance of threat. We also calculated threat interference scores for the baseline phase and the test phase of the dot probe task by subtracting the mean reaction time on neutral trials (i.e., the trials in which two neutral pictures were used as cues) from the mean reaction time on trials with a threatening picture (i.e., the congruent and incongruent trials). Positive interference scores indicate more interference on trials with a threatening picture than on trials with two neutral pictures.

For the interference task, we also removed trials with errors $(3.54 \%)$, latencies faster than $150 \mathrm{~ms}$ and slower than $1500 \mathrm{~ms}(0.47 \%)$, and latencies deviating more than three $S D$ s from each individuals' mean (1.71\%). From the remaining data, we calculated 
interference scores by subtracting the mean reaction time on neutral trials from the mean reaction time on threatening trials. Scores larger than zero indicate interference by arousing pictures.

Attentional training effects. We conducted a 2 (Experiment Phase: pre-training versus post-training) by 2 (Attention Group: attend threat versus avoid threat) repeated measures ANOVA on the attentional bias scores. This analysis yielded a marginally significant main effect of Attention Group, $F(1,52)=3.13, p=.08$. More importantly, the interaction was also significant, $F(1,52)=16.26, p<.001$ (see Figure 1). Follow-up analyses showed that, before the training phase, the attend threat group tended to avoid the threatening pictures relative to the avoid threat group, $F(1,52)=3.49, p=.07$, Cohen's $d=0.51 .^{2}$ In this phase, the attentional bias score of the attend threat group was significantly smaller than zero, $t(25)=2.12, p<.05$, whereas the attentional bias score of the avoid threat group did not differ from zero, $t(27)<1, p=.64$. After the training phase, participants in the attend threat group oriented significantly more to the threatening pictures compared to participants in the avoid threat group, $F(1,52)=$ $14.56, p<.001$, Cohen's $d=1.04$. Participants in the attend threat group showed a marginally significant attentional bias towards threat, $t(25)=1.81, p=.08$, whereas participants in the avoid threat group showed attentional avoidance of threat, $t(27)=$ $3.53, p<.005$. Finally, the attend threat group showed a significant increase in attentional bias from pre- to post-training, $F(1,25)=7.92, p<.01$, Cohen's $f=0.56$, and the avoid threat group showed a significant decrease in attentional bias from pre- to post-training, $F(1,27)=8.54, p<.01$, Cohen's $f=0.56 .{ }^{3}$ A 2 (Experiment Phase) x 2 (Attention Group) repeated measures ANCOVA with state and trait anxiety at the beginning of the experiment as covariates did not change this pattern of results. In sum, 
these data show that we successfully induced an attentional bias towards threat in the attend threat group, and we successfully induced attentional avoidance of threat in the avoid threat group.

Effects of attention training on emotional interference. Our design allowed us to investigate the effect of the attention training on emotional interference in both the dot probe task and the interference task. First, for the dot probe task, we entered the interference scores in a 2 (Experiment Phase: pre-training versus post-training) x 2 (Attention Group: attend threat versus avoid threat) repeated measures ANOVA. There were no significant main effects, both $F \mathrm{~s}<1$, but the interaction was marginally significant, $F(1,52)=3.12, p=.084$. However, this interaction was mainly driven by a marginally significant difference between the two groups in the baseline phase $\left(M_{\text {attend }}=\right.$ $\left.16.19, S D=22.91 ; M_{\text {avoid }}=3.82, S D=23.29\right), F(1,52)=3.86, p=.055$, Cohen's $d=$ 0.54. In the test phase, there was no significant difference between the two groups $\left(M_{\text {attend }}=4.12, S D=30.98 ; M_{\text {avoid }}=9.04, S D=20.41\right), F(1,52)<1$. Comparisons of the two experiment phases revealed that neither the decrease in interference in the attend threat group nor the increase in interference in the avoid group were significant, both $F \mathrm{~s}$ $<1.99$, both $p$ s $>.17$.

Next, we conducted a univariate ANOVA on the interference scores as measured in the interference task with Attention Group as a between subjects factor. This analysis did not reveal a significant difference between the two groups, $F(1,52)<1, p=.37$, Cohen's $d=0.25$. One-sample $t$-tests showed that both the attend threat group, $t(25)=$ $5.53, p<.001$, and the avoid threat group, $t(27)=3.65, p<.005$, responded slower to targets when they were preceded by a threatening picture compared to when they were preceded by a neutral picture (see Figure 2). Entering state and trait anxiety before the 
beginning of the experiment as covariates in the analyses did not change the pattern of results. Finally, the interference score of the dot probe test phase was significantly correlated with the interference score of the interference task, $r=.33, p<.05$. In sum, both attention groups experienced more interference by threatening pictures compared to neutral pictures, but the attention training had no effect on the magnitude of the interference effect.

Effects of attention training on self reported appraisal of pictures. Overall, the threatening pictures were rated as more threatening, $F(1,53)=157.27, p<.001$, more arousing, $F(1,53)=92.30, p<.001$, and more negative, $F(1,53)=92.58, p<.001$, than the neutral pictures. There were no differences between the two attention groups on any of the ratings of the threatening pictures, all $F \mathrm{~s}<1.02$, all $p \mathrm{~s}>.31$ (see Table 1). Similar analyses but now separately for the set that we used in the dot probe task and the set that we used in the interference task again showed no differences between the two groups on any of the picture appraisal variables, all $F \mathrm{~s}<1.03$, all $p \mathrm{~s}>.31$. However, overall, the threatening pictures that were used in the dot probe task were rated as more arousing, $F(1,52)=4.36, p<.05$, more threatening, $F(1,52)=4.18, p<.05$, and more negative, $F(1,52)=3.91, p=.053$, than the pictures that were used in the interference task. Finally, a 2 (Attention Group) x 2 (Picture Type: neutral versus threatening) repeated measures ANOVA on the viewing times revealed only a main effect of Picture Type, $F(1,52)=51.24, p<.001 ;$ all other $F \mathrm{~s}<1$. As expected, participants looked longer at threatening pictures $(M=2419 \mathrm{~ms}, S D=1111)$ than neutral pictures $(M=1784 \mathrm{~ms}, S D$ $=717$ ). Hence, our data show that the attentional training had no impact on the appraisal of threatening stimuli.

\section{Discussion}


According to several authors, training anxious or fearful individuals to attend away from threat is promising therapeutic tool to reduce anxiety (MacLeod, Koster, \& Fox, 2009). More specifically, attentional bias reduction could be an easy to administer and cheap first line intervention. In the current study we tested the implicit assumption underlying attentional training research that the attentional changes obtained through the typically used modified dot probe task are not a reflection of mere practice in the dot probe task, but that these attentional changes also transfer to other attention tasks and thus fundamentally change attentional processing in everyday life. Despite the successful training of attentional bias towards and away from threat as measured with a standard dot probe task, training showed no transfer to levels of emotional interference (either measured within the dot probe task or on a subsequent interference task), nor did it influence self-rated stimulus properties.

As the absence of any effects of the attentional training on the emotional interference task is unexpected, the validity of this null-finding should be considered. First, it is possible that our training manipulation was not strong enough to produce the expected pattern of results. Contrary to this explanation, both groups showed a substantial change in attentional bias in the expected direction from pre- to post-training as measured with the dot probe task, with Cohen's $d=0.55$ for both groups. These results clearly show that we induced changes in attentional bias in the dot probe task, and thus that our training manipulation was relatively strong.

There are at least two different explanations for why this strong training effect did not transfer to the interference task. First, we used a different picture set for the emotional interference task than for the attention training. It is possible that the training manipulation was only effective in changing the allocation of attention for the pictures 
that were used in the dot probe task, but not in changing attention for the new pictures that were used in the interference task. However, several studies have shown that attentional retraining transfers to new stimulus materials (e.g., Amir, Beard, Burns et al., 2009). Thus, though possible, it seems unlikely that the absence of group differences in the interference task is caused by the use of a new set of stimuli. Second, it is possible that the attentional training did not transfer to the interference task because the dot probe task involves the spatial allocation of attention, whereas the interference task has no such spatial component. The emotional interference effect is driven by a difficulty to disengage attention away from the semantic content of certain stimuli (e.g., Fox, Russo, Bowles, \& Dutton, 2001), whereas attentional bias as measured with the dot probe task is mainly based on a difficulty to disengage attention away from the spatial location of threatening stimuli (Koster, Crombez, Verschuere, \& De Houwer, 2004). Thus, it is possible that the effects of attentional training with the dot probe task are limited to spatial attention, and that the effect fails to generalize to other components of attention. However, we acknowledge that our study offers only a first attempt to investigate the transfer of the attentional training effect in the dot probe task to a non-spatial attention task. Future research in which other measures of attentional bias are used, such as for instance the emotional Stroop paradigm or the attentional blink task, are needed in order to further address the question of transfer to other tasks. Bearing this limitation in mind, a lack of generalization across different sets of stimuli or attentional processes does, however, pose a challenge for the dominant theories of attentional bias modification. According to these theories, in order for attention training to influence attention in the real world, training should generalize to both different stimulus materials and different components of attention, and thus different measures of attention. 
The present data point to an important hiatus in our knowledge on the attentional mechanisms affected by attentional retraining (see also Koster, Baert, Bockstaele, \& De Raedt, 2010), and they imply that future work should further improve upon the existing procedures to train attention. It is noteworthy that the current version of the dot probe training has been developed mainly because the dot probe task is a widely used measure of attentional bias (Mathews \& MacLeod, 2002). From a training perspective, several modifications of current attentional retraining procedure could be useful to optimize the transfer of training to different tasks and situations. First, a training procedure could incorporate training on multiple components of attention in different types of visual displays, instead of the fixed stimulus presentation format in the current training. Training procedures could also be presented to different modalities to facilitate crossmodal transfer of training. Finally, training conditions could be dynamically adjusted based on individual performance. Hence, given the applied potential of attentional retraining (e.g., Bar-Haim, 2010), the investigation of optimal training procedures is a promising area of research.

Our study has several limitations. For instance, in line with other research (e.g., Amir et al., 2008; Hazen et al., 2009), we chose to present the cue pictures in the dot probe task for $500 \mathrm{~ms}$. Recently, Staugaard (2010) has argued that this presentation duration of $500 \mathrm{~ms}$ might be suboptimal, and that the measurement of attentional bias at this presentation duration becomes less reliable. Also, our experiment was conducted with a non-anxious student sample. Further research with (clinically) anxious individuals is needed to investigate whether our observed lack of transfer can be replicated in such populations. Another limitation concerns the difference in attentional bias between the two groups in the pre-training assessment phase. As we assigned 
participants randomly to one of the two groups, this baseline difference is hard to explain. However, it is important to note that both groups showed a change in attentional bias that was in line with the training manipulation. That is, participants in the attend threat group had a higher attentional bias towards threat after the training, whereas participants in the avoid threat training group showed a decrease in attentional bias from pre- to post-training. A more fundamental limitation concerns the possibility that our study lacked the statistical power to capture the possible transfer of these effects to the interference task. Using G*Power 3 (Faul, Erdfelder, Lang, \& Buchner, 2007), we calculated the magnitude of effect sizes that we should have detected, given our sample size and the conventional value of .80 for minimal statistical power. This analysis showed that our sample was only large enough to detect a relatively large difference, with Cohen's $d=0.78$. Hence, it is well possible that our sample was not large enough to detect small to moderate effects. We also did not include a pre-training assessment of emotional interference, nor did we ask participants to rate the arousal, valence and threat value of the pictures before the training. Therefore, it is possible that both groups showed a similar decrease in emotional interference and a similar decrease in ratings of arousal, negative valence and threat value of the stimuli. A final limitation is the absence of a no-training control group. If both attentional manipulations had similar effects on the amount of interference, these effects should appear in comparison with such a no-training control group. Although at first sight it may seem contradictory that the two opposite attentional manipulations have the same effect (e.g., MacLeod et al., 2002), such data have been reported before. For instance, Klumpp and Amir (2010) trained socially anxious participants to either attend to or avoid threat, and compared their levels of state anxiety in response to a social stressor with a no-training control 
group. Interestingly, they found that both the attend threat group and the avoid threat group showed a smaller increase in state anxiety compared to the no-training control group in response to the social stress task. In sum, the absence of a no-training control group and a pre-training assessment phase complicates the interpretation of the data of our current experiment. It is both possible that neither manipulation of attention had an effect on the level of interference and the appraisal of the stimuli, and that both manipulations had the same effect. Therefore, our study does not allow for strong conclusions with respect to the influence of changes in attention on the assessment of the arousal, valence and threat value of stimuli. However, our data do suggest that training individuals to disengage their attention away from threat in a dot probe task does not necessarily transfer to measures of emotional interference. 
Acknowledgements

Preparation of this paper was supported by Grant BOF/GOA2006/001 of Ghent University. The first author would like to thank prof. dr. J. Slabbert and P. Beukes from iThemba Labs for their assistance in finalizing this manuscript. 


\section{References}

Amir, N., Weber, G., Beard, C., Bomyea, J., \& Taylor, C. T. (2008). The effect of a single-session attention modification program on response to a public-speaking challenge in socially anxious individuals. Journal of Abnormal Psychology, 117, 860-868.

Amir, N., Beard, C., Burns, M., \& Bomyea, J. (2009). Attention modification program in individuals with generalized anxiety disorder. Journal of Abnormal Psychology, 118, 28-33.

Amir, N., Beard, C., Taylor, C. T., Klumpp, H., Elias, J., Burns, M., \& Chen, X. (2009). Attention training in individuals with generalized social phobia: A randomized controlled trial. Journal of Consulting and Clinical Psychology, 77, 961-973.

Bar-Haim, Y. (2010). Research review: Attention bias modification (ABM): A novel treatment for anxiety disorders. Journal of Child Psychology and Psychiatry, 51, 859-870.

Beck, A. T., Emery, G., \& Greenberg, R. (1985). Anxiety disorders and phobias: A cognitive perspective. New York: Basic Books.

Cohen, J. (1992). A power primer. Psychological Bulletin, 112, 155-159.

De Houwer, J., \& Tibboel, H. (2010). Stop what you are not doing! Emotional pictures interfere with the task not to respond. Psychonomic Bulletin \& Review, 17, 699703.

Eysenck, M. W. (1992). Anxiety: The cognitive perspective. Hove: Erlbaum.

Fox, E., Russo, R., Bowles, R., \& Dutton, K. (2001). Do threatening stimuli draw or hold visual attention in subclinical anxiety? Journal of Experimental Psychology: General, 130, 681-700. 
Hakamata, Y., Lissek, S., Bar-Haim, Y., Britton, J. C., Fox, N. A., Leibenluft, E., Ernst, M., et al. (2010). Attention bias modification treatment: A meta-analysis toward the establishment of novel treatment for anxiety. Biological Psychiatry, 68, 982990.

Hazen, R. A., Vasey, M. W., \& Schmidt, N. B. (2009). Attentional training: A randomized clinical trial for pathological worry. Journal of Psychiatric Research, 43, 627-633.

Inquisit 2.0 [Computer software]. (2007). Seattle, WA: Millisecond Software.

Klumpp, H., \& Amir, N. (2010). Preliminary study of attention training to threat and neutral faces on anxious reactivity to a social stressor in social anxiety. Cognitive Therapy and Research, 34, 263-271.

Koster, E.H.W., Baert, S., Bockstaele, M., \& De Raedt, R. (2010). Attentional retraining procedures: Manipulating early or late components of attentional bias? Emotion, 10. 230-236.

Koster, E. H. W., Crombez, G., Verschuere, B., \& De Houwer, J. (2004). Selective attention to threat in the dot probe paradigm: Differentiating vigilance and difficulty to disengage. Behaviour Research and Therapy, 42, 1183-1192.

Lang, P. J., Bradley, M. M., \& Cuthbert, B. N. (2005). International affective picture system (IAPS): Instruction manual and affective ratings. Technical Report A-6, The Center for Research in Psychophysiology, University of Florida.

Lang, P. J., Greenwald, M. K., Bradley, M. M., \& Hamm, A. O. (1993). Looking at pictures: Affective, facial, visceral, and behavioral reactions. Psychophysiology, $30,261-273$ 
Li, S., Tan, J., Qian, M., \& Liu, X. (2008). Continual training of attentional bias in social anxiety. Behaviour Research and Therapy, 46, 905-912.

MacLeod, C., Koster, E. H. W., \& Fox, E. (2009). Whither cognitive bias modification research? Commentary on the special section articles. Journal of Abnormal Psychology, 118, 89-99.

MacLeod, C., Mathews, A., \& Tata, P. (1986). Attentional bias in emotional disorders. Journal of Abnormal Psychology, 95, 15-20.

MacLeod, C., Rutherford, E., Campbell, L., Ebsworthy, G., \& Holker, L. (2002). Selective attention and emotional vulnerability: Assessing the causal basis of their association through the experimental manipulation of attentional bias. Journal of Abnormal Psychology, 111, 107-123.

Mathews, A., \& MacLeod, C. (2002). Induced processing biases have causal effects on anxiety. Cognition and Emotion, 16, 331-354.

Mogg, K., \& Bradley, B. P. (1998). A cognitive-motivational analysis of anxiety. Behaviour Research and Therapy, 36, 809-848.

Mogg, K., Holmes, A., Garner, M., \& Bradley, B. P. (2008). Effects of threat cues on attentional shifting, disengagement and response slowing in anxious individuals. Behaviour Research and Therapy, 46, 656-667.

Rachman, S. (1998). Anxiety. Hove, UK: Psychology Press Ltd.

Reese, H. E., McNally, R. J., Najmi, S., \& Amir, N. (2010). Attention training for reducing spider fear in spider-fearful individuals. Journal of Anxiety Disorders, $24,657-662$.

Schimmack, U. (2005). Attentional interference effects of emotional pictures: Threat, negativity, or arousal? Emotion, 5, 55-66. 
Schmidt, N. B., Richey, J. A., Buckner, J. D., \& Timpano, K. R. (2009). Attention training for generalized social anxiety disorder. Journal of Abnormal Psychology, 118, 5-14.

Spielberger, C. D., Gorsuch, R. L., Lushene, R., Vagg, P. R., \& Jacobs, G. A. (1983). Manual for the State-Trait Anxiety Inventory. Palo Alto, CA: Consulting Psychologists Press.

Staugaard, S. R. (2010). Threatening faces and social anxiety: A literature review. Clinical Psychology Review, 30, 669-690.

Van Bockstaele, B., Verschuere, B., Koster, E. H. W., Tibboel, H., De Houwer, J., \& Crombez, G. (2011). Effects of attention training on self-reported, implicit, physiological and behavioural measures of spider fear. Journal of Behavior Therapy and Experimental Psychiatry, 42, 211-218.

van der Ploeg, H. M., Defares, P. B., \& Spielberger, C. D. (1980). Handleiding bij de Zelf-Beoordelings Vragenlijst. [Manual for the Dutch adaptation of the STAI-Y]. Lisse: Swets \& Zeitlinger.

Verschuere, B., Crombez, G., \& Koster, E. H. W. (2001). The international affective picture system: A Flemish validation study. Psychologica Belgica, 41, 205-217.

Williams, J. M. G., Watts, F. N., MacLeod, C., \& Mathews, A. (1997). Cognitive psychology and the emotional disorders (2nd ed.). New York: Wiley. 
Figure captions

Figure 1. Attentional Bias Scores as a Function of Experiment Phase and Attention Group.

Figure 2. Reaction Times in the Interference Task as a Function of Cue Picture and Attention Group. 


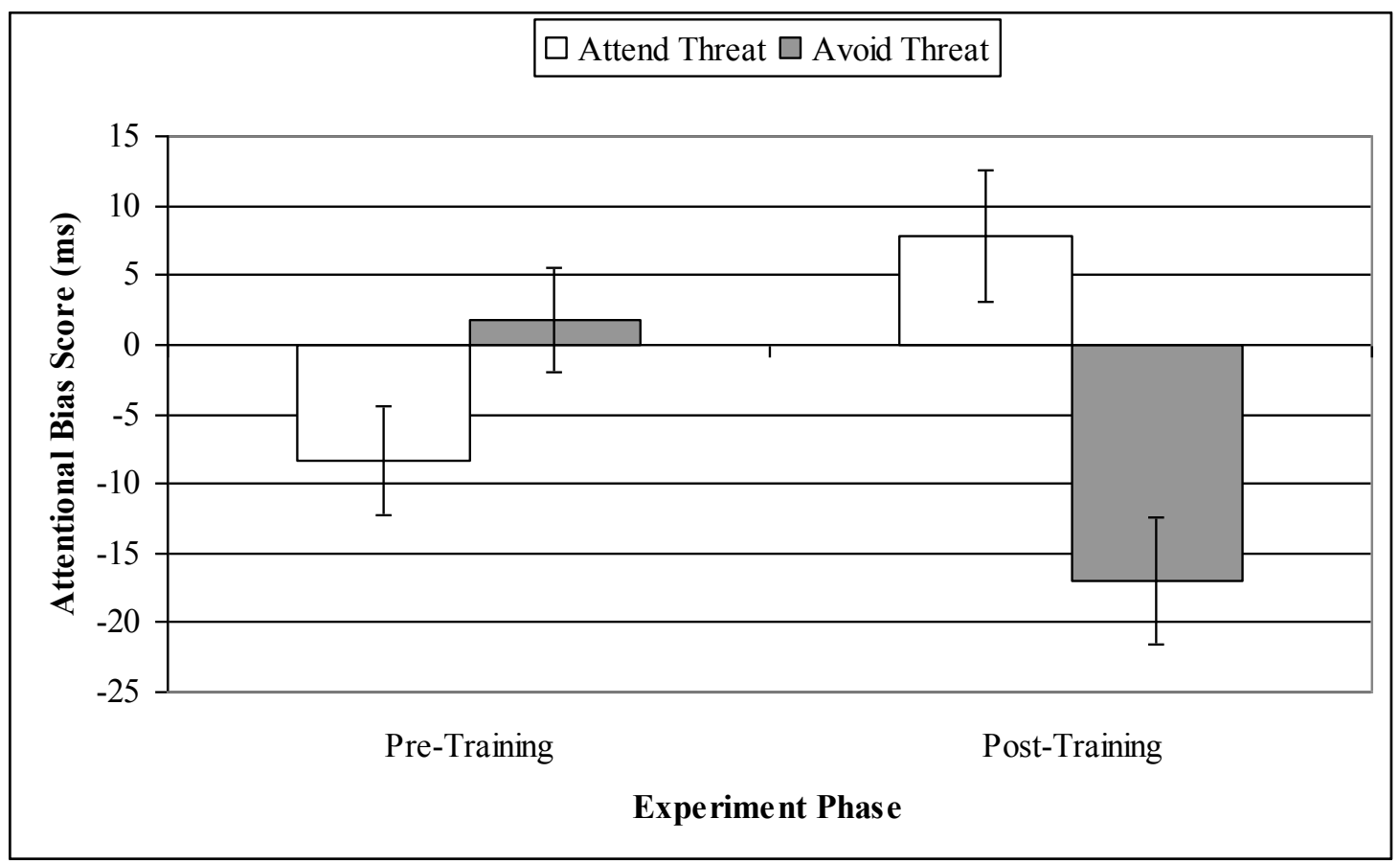

Figure 1. 


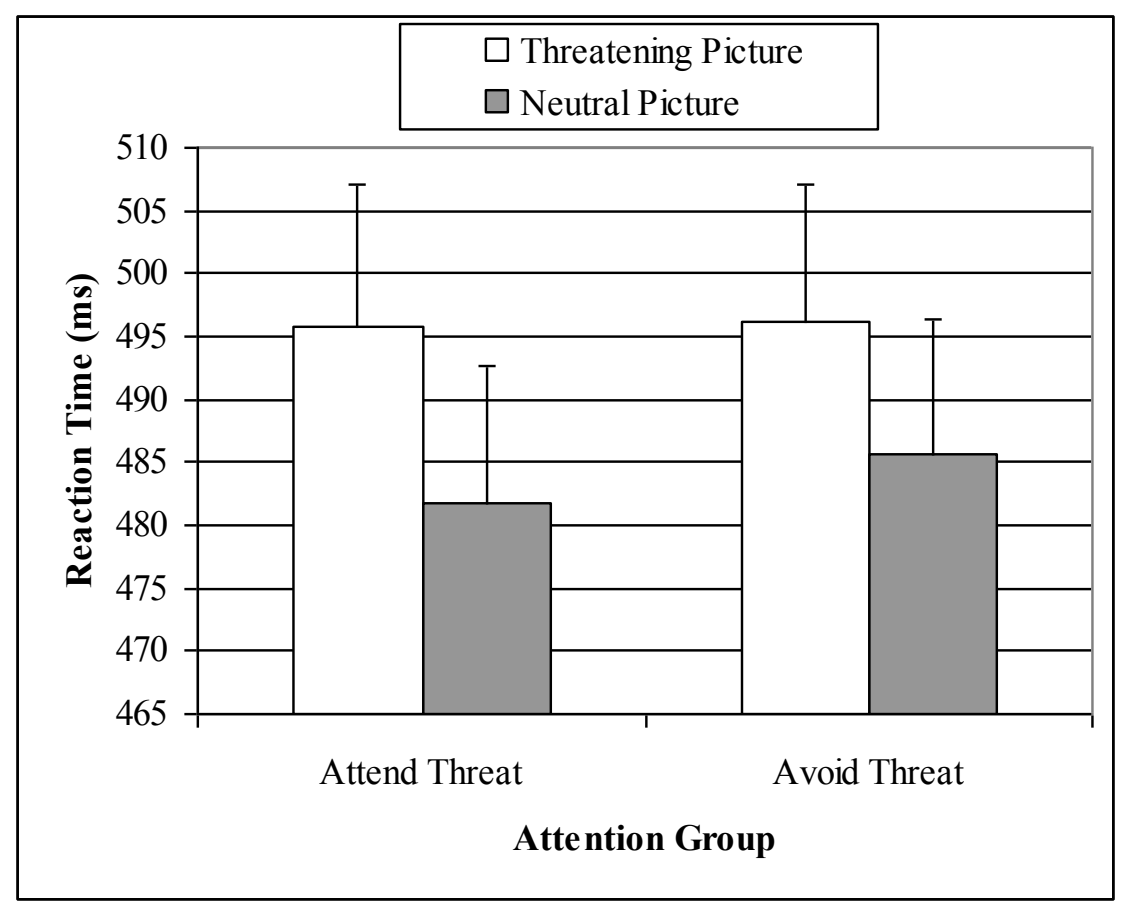

Figure 2. 
Table 1. Explicit Arousal, Valence and Threat Ratings of all Threatening Pictures Used in the Experiment, and the Effect Size of the Difference between the Two Groups.

\begin{tabular}{|c|c|c|c|c|c|}
\hline & \multicolumn{2}{|c|}{ Attend threat } & \multicolumn{2}{|c|}{ Avoid threat } & \multirow[t]{2}{*}{ Cohen's $d$} \\
\hline & $M$ & $S E$ & $M$ & $S E$ & \\
\hline Arousal total & 4.38 & 0.41 & 3.97 & 0.40 & 0.19 \\
\hline Dot probe task & 4.52 & 0.43 & 4.05 & 0.41 & 0.21 \\
\hline Interference task & 4.24 & 0.41 & 3.89 & 0.40 & 0.17 \\
\hline Valence total & 6.46 & 0.34 & 6.25 & 0.33 & 0.12 \\
\hline Dot probe task & 6.54 & 0.35 & 6.34 & 0.34 & 0.11 \\
\hline Interference task & 6.39 & 0.35 & 6.15 & 0.33 & 0.13 \\
\hline Threat total & 5.37 & 0.43 & 4.76 & 0.42 & 0.27 \\
\hline Dot probe task & 5.50 & 0.45 & 4.86 & 0.44 & 0.28 \\
\hline Interference task & 5.24 & 0.43 & 4.66 & 0.41 & 0.26 \\
\hline
\end{tabular}




\section{Footnotes}

1. The following IAPS pictures were used in the experiment: threat subset 1 : $1050,1201,1930,6260,6350,6550$; threat subset 2: 1120, 1300, 6230, 6313, 6540, 6560; neutral subset 1: 5510, 7006, 7090, 7150, 7190, 7234; neutral subset 2: 7002, 7009, 7140, 7175, 7224, 7550; neutral practice: 5720, 5740, 6150, 7000, 7050, 7080.

2. Effect sizes of group differences were estimated with Cohen's $d$. According to Cohen (1992), values around 0.20 represent small effects, values around 0.50 represent medium effects and values of 0.80 and larger represent large effects. Effect sizes for within-group differences and interactions were estimated using Cohen's $f$, with values from 0.10 representing small effects, values from 0.25 representing medium effects and values from 0.40 representing large effects (Cohen, 1992). We calculated $f$ using the following formula: $f=\sqrt{ }\left[\eta_{\mathrm{p}}{ }^{2} /\left(1-\eta_{\mathrm{p}}^{2}\right)\right]$.

3. Overall, the neutral pictures $\left(M=96.09 \mathrm{~cd} / \mathrm{m}^{2}, S D=34.62\right.$, range $=41.87 \mathrm{~cd} / \mathrm{m}^{2}$ $\left.-150.46 \mathrm{~cd} / \mathrm{m}^{2}\right)$ had a marginally higher luminance than the threatening pictures $(M=$ $68.01 \mathrm{~cd} / \mathrm{m}^{2}, S D=35.95$, range $\left.=31.51 \mathrm{~cd} / \mathrm{m}^{2}-156.21 \mathrm{~cd} / \mathrm{m}^{2}\right), F(1,22)=3.80, p=$ .067. In order to investigate whether the difference in luminance between the neutral and the threatening pictures had a profound effect on our data, we removed all trials containing one of the two neutral pictures with the highest luminance and all trials containing one of the two threatening pictures with the lowest luminance levels from the data set. As a result, threatening and neutral pictures did no longer differ with regard to their luminance, $F(1,18)<1$. A repeated measures ANOVA on the attentional bias scores from the remaining data set with Experiment Phase (pre-training versus posttraining) as a within subjects factor, and Training Group (attend threat versus avoid threat) as a between subjects factor revealed a significant interaction between 
Experiment Phase and Attention Group, $F(1,52)=11.61, p<.005$. Follow-up contrast comparisons showed that participants in the attend threat group attended more to threat after the training $(M=5.09, S D=28.18)$ than before the training $(M=-12.89, S D=$ 28.05), $F(1,25)=6.91, p<.05$. Participants in the avoid threat training group avoided threat more following the training $(M=-17.73, S D=31.43)$ compared to before the training $(M=1.74, S D=28.82), F(1,27)=5.28, p<.05$. Overall, these results closely mirror the results of the analyses on the complete data set, and they show that the differences in luminance between the threatening and neutral pictures are unlikely to account for our general pattern of findings. 